\title{
Transverse Bragg resonance laser amplifier
}

\author{
Amnon Yariv, Yong Xu, and Shayan Mookherjea \\ Department of Applied Physics, 128-95, California Institute of Technology, Pasadena, California 91125
}

Received August 9, 2002

\begin{abstract}
We propose and analyze a new type of optical amplifier that is formed by addition of gain in the periodic cladding of a transverse Bragg resonance waveguide [Opt. Lett. 27, 936 (2002)]. Using the coupled-wave formalism, we calculate the mode profiles, the exponential gain constant, and, for comparison, the gain enhancement compared with those of conventional semiconductor optical amplifiers. In contrast with coupled-mode theory, in one-dimensional structures (e.g., the distributed-feedback laser) the exponential gain constant in the longitudinal direction is involved in both longitudinal and transverse confinement, and its solution has to be achieved self-consistently, together with the quantized guiding channel width. (C) 2003 Optical Society of America

OCIS codes: $130.2790,140.3280,130.3120,140.3490,230.7400,250.4480$
\end{abstract}

A new type of optical laser (amplifier or oscillator) is proposed and analyzed. The amplifier consists of a guiding channel (the core), which is sandwiched between two periodic-index media (the cladding), or, in a cylindrical geometry, is surrounded by the periodic-index medium. The periodic media possess optical gain; the core may or may not (both cases are analyzed).

The basic concept behind this amplifier is as follows: It is well known that one can form optical waveguides that depend on Bragg reflection from a periodic cladding rather than on total internal reflection. ${ }^{1,2}$ It is also known that, if the periodic medium possesses optical gain, an incident wave can be amplified on reflection. ${ }^{3,4}$ We combine these two facts and, referring to Fig. 1, imagine a planelike wave zigzagging inside a uniform guiding channel that is flanked on each side by an amplifying periodic medium. In as much as the wave is amplified on each reflection, we expect the wave to grow exponentially along $z$.

In conventional laser amplifiers, one usually solves for the electromagnetic modes of the passive confining structure and adds the optical gain, assuming implicitly (and most of the time justifiably) that the presence of gain modifies the modes only negligibly. Such is not the case when the confinement is due to Bragg reflection, because then the field distribution inside the periodic medium changes drastically when gain is present. The amplification and confinement issues have to be addressed self-consistently. As expected, the theoretical issues that arise and some of the features of the transverse Bragg resonance amplifiers are novel.

Referring to Fig. 1, we seek a modal propagating solution to the Helmholtz equation

$$
\nabla^{2} \mathbf{E}(\mathbf{r}, t)-\mu \epsilon(\mathbf{r}) \frac{\partial^{2} \mathbf{E}(\mathbf{r}, t)}{\partial t^{2}}=0
$$

We allow $\epsilon(\mathbf{r})$ to be complex, $\epsilon(\mathbf{r})=\epsilon_{R}(x)+i \epsilon_{I}(x)$, and seek a solution with an $\exp [i(\omega t-\beta z)]$ dependence, where $\beta \equiv \beta_{R}+i \beta_{I}$. Modal amplification obtains when $\beta_{I}>0$.
The wave equation, Eq. (1), now reads as

$$
\frac{\mathrm{d}^{2} E}{\mathrm{~d} x^{2}}+\left(\omega^{2} \mu \epsilon_{R}-\beta_{R}^{2}\right) E+i\left(\omega^{2} \mu \epsilon_{I}-2 \beta_{R} \beta_{I}\right) E=0,
$$

where we have assumed that $\left|\beta_{I}\right| \ll\left|\beta_{R}\right|$.

In the periodic cladding, with unit cell $(b \hat{\mathbf{x}} \times a \hat{\mathbf{z}})$, we can represent

$$
\epsilon_{R}(\mathbf{r})=\sum_{m, n} \epsilon_{R m n} \exp \left(i \mathbf{K}_{m n} \cdot \mathbf{r}\right),
$$

where

$$
\mathbf{K}_{m n}=m(2 \pi / b) \hat{\mathbf{x}}+n(2 \pi / a) \hat{\mathbf{z}} .
$$

The only terms in the expansion that are capable of coupling, by phase-matched Bragg reflection, the two plane-wave components of the field in the core region are the terms $m=1$ and $n=0$, which can be written $\mathrm{as}^{5}$

$$
\epsilon_{R}(x)=\epsilon_{R 0}-2 \epsilon_{1} \cos \left(\frac{2 \pi}{b} x\right)
$$

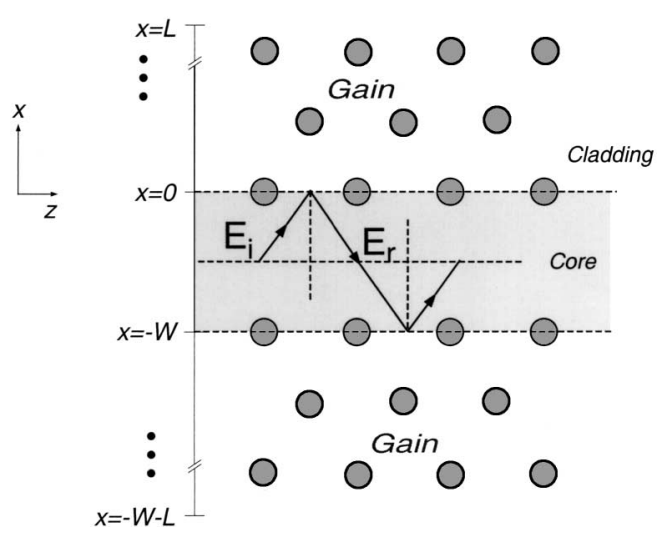

Fig. 1. Top view of a two-dimensional periodic waveguiding structure: a guiding channel of width $W$ (core) between two semi-infinite arrays of air holes in a periodic pattern, e.g., a triangular lattice (cladding). Also shown, in the core, are the two in-plane $k$-vectors of plane waves $E_{\mathrm{i}}$ and $E_{\mathrm{r}}$ that compose the waveguide mode. 
where

$$
\epsilon_{1} \equiv \epsilon_{01}=\frac{1}{a b} \iint_{\text {u.c. }} \epsilon(\mathbf{r}) \exp \left(-i \frac{2 \pi}{b} x\right) \mathrm{d} x \mathrm{~d} z
$$

and u.c. means unit cell. A substitution of Eq. (4) into Eq. (2) allows Eq. (2) to be written as

$$
\begin{aligned}
\frac{\mathrm{d}^{2} E}{\mathrm{~d} x^{2}}+k_{0}^{2} E-2 \omega^{2} \mu \epsilon_{1} \cos \left(\frac{2 \pi}{b} x\right) E \\
+i\left(\omega^{2} \mu \epsilon_{I}-2 \beta_{R} \beta_{I}\right) E=0,
\end{aligned}
$$

where $k_{0}^{2} \equiv \omega^{2} \mu \epsilon_{R 0}-\beta_{R}{ }^{2}$.

We look for solutions in the combined core-pluscladding regions of the general form

$$
\begin{aligned}
E(z, x, t)= & {\left[A(x) \exp \left(-i k_{0} x\right)+B(x) \exp \left(i k_{0} x\right)\right] } \\
& \times \exp [i(\omega t-\beta z)] \\
\equiv & E_{\perp}(x) \exp [i(\omega t-\beta z)]
\end{aligned}
$$

our search is motivated by the fact that, when $\epsilon_{1}=0$ and $\epsilon_{I}=0$ (which implies that $\beta_{I}=0$ ), $A$ and $B$ are independent of $x$ and also of each other. A substitution of Eq. (7) into Eq. (6) and in the vicinity of the Bragg condition $k_{0} \approx \pi / b\left(k_{0} \approx 2 \pi / b\right.$ for the triangular lattice) results in the familiar coupled-wave equations ${ }^{6}$

$$
\begin{aligned}
& \frac{\mathrm{d} A}{\mathrm{~d} x}=\gamma A+\kappa^{*} B \exp \left[i 2\left(k_{0}-\pi / b\right) x\right], \\
& \frac{\mathrm{d} B}{\mathrm{~d} x}=-\gamma B+\kappa A \exp \left[-i 2\left(k_{0}-\pi / b\right) x\right]
\end{aligned}
$$

on the interval $0<x \leq L$, the cladding region. We have defined

$$
\gamma=\frac{\omega^{2} \mu \epsilon_{I}^{(\mathrm{clad})}}{2 k_{0}}-\frac{\beta_{R} \beta_{I}}{k_{0}}, \quad \kappa=-\frac{i \omega^{2} \mu \epsilon_{1}}{2 k_{0}} .
$$

We solve Eqs. (8) subject to the condition that at the outer edge of the cladding there is no reflected wave; i.e., that $B(L)=0$. The result is a superposition of Bloch waves:

$$
\begin{aligned}
& E_{\perp}{ }^{(\text {clad })}(x)=F\{\exp (-i \pi x / b) \\
& \quad \times \frac{(\gamma-i \Delta k) \sinh [S(L-x)]-S \cosh [S(L-x)]}{(\gamma-i \Delta k) \sinh (S L)-S \cosh (S L)} \\
& \left.\quad+\exp (+i \pi x / b) \frac{\kappa \sinh [S(L-x)]}{(\gamma-i \Delta k) \sinh (S L)-S \cosh (S L)}\right\},
\end{aligned}
$$

where

$$
S \equiv\left[|\kappa|^{2}+(\gamma-i \Delta k)^{2}\right]^{1 / 2}, \quad \Delta k \equiv k_{0}-(\pi / b),
$$

and $F$ is a scale factor that will be needed in matching the fields at $x=0$. The exact Bloch solution [Eq. (10)] is a key ingredient in our modal solution.

In the uniform-core region $(-W \leq x \leq 0)$ the field is governed by the Helmholtz equation [Eq. (6)], with $\epsilon_{1}=0$. We assume that the real part of the average dielectric constant $\epsilon_{R}$ is the same in both the core and the cladding regions. We present results for two cases: gain in the cladding as well as in the core, $\epsilon_{I}{ }^{(\text {core })}=\epsilon_{I}{ }^{\text {(clad) }}$; and gain in the cladding only,
$\epsilon_{I}^{(\text {core })}=0 . \quad$ We write the solution as

$$
E_{\perp}{ }^{(\text {core })}(x)=\exp \left[-i k^{\prime}(x+W / 2)\right] \pm \exp \left[i k^{\prime}(x+W / 2)\right],
$$

where

$$
k^{\prime} \equiv k_{0}\left[1+\frac{i}{2 k_{0}^{2}}\left(\omega^{2} \mu \epsilon_{I}^{(\text {core })}-2 \beta_{R} \beta_{I}\right)\right] .
$$

The signs + and - in Eq. (12) refer to modes of even and odd symmetry, respectively.

In Eqs. (10) and (12) we have the field solutions for the cladding and the core, respectively. We stitch these two solutions together at the interface by requiring that the $+x$-traveling component in the core be the same as its counterpart in the cladding at $x=0$. The same condition is applied to the waves traveling in the $-x$ direction. This ensures the continuity of the total fields at $x=0$, and, to a very high degree (consistent with the basic nature of the perturbation involved in a coupled-wave approach), the continuity of the total field derivative. The same condition is used to calculate the reflectance of Bragg gratings. ${ }^{6}$ These two conditions can be expressed, with Eqs. (10) and (12), as

$$
\begin{aligned}
\exp \left(-i k^{\prime} \frac{W}{2}\right) & =F, \\
\exp \left(i k^{\prime} \frac{W}{2}\right) & =\frac{ \pm \kappa F}{(\gamma-i \Delta k)-S \operatorname{coth}(S L)} .
\end{aligned}
$$

It follows that

$$
\exp \left(i k^{\prime} W\right)=\frac{ \pm \kappa}{(\gamma-i \Delta k)-S \operatorname{coth}(S L)},
$$

where

$$
\begin{aligned}
S & \equiv\left[|\kappa|^{2}+(\gamma-i \Delta k)^{2}\right]^{1 / 2}, \\
\gamma & =\frac{\omega^{2} \mu \epsilon_{I}^{(\mathrm{clad})}}{2 k_{0}}-\frac{\beta_{R} \beta_{I}}{k_{0}}, \\
\kappa & =-\frac{i \omega^{2} \mu \epsilon_{1}}{2 k_{0}}, \quad k_{0}^{2} \equiv \omega^{2} \mu \epsilon_{R 0}-\beta_{R}{ }^{2}, \\
k^{\prime} & \equiv k_{0}\left[1+\frac{i}{2 k_{0}^{2}}\left(\omega^{2} \mu \epsilon_{I}^{(\text {core })}-2 \beta_{R} \beta_{I}\right)\right] .
\end{aligned}
$$
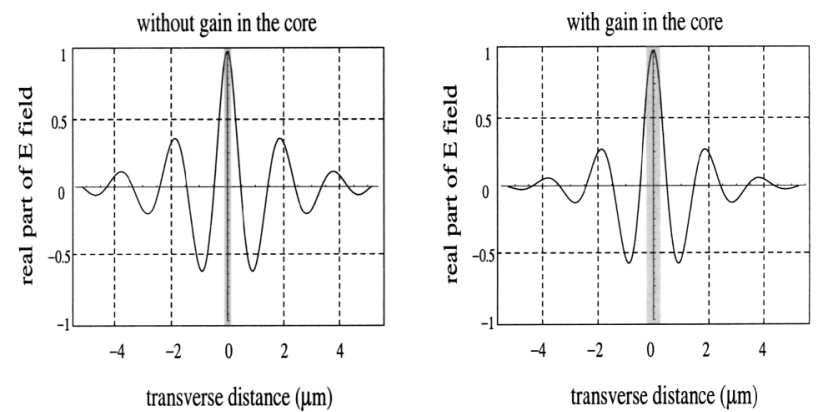

Fig. 2. Transverse $(x)$ modal profile, with the shaded region indicating the core width, for $L=5 \mu \mathrm{m}$ and $\epsilon_{I}^{\text {(core) }}=$ $\epsilon_{I}{ }^{(\mathrm{clad})}$. Two structures are considered: for the figure at the left there is no gain in the core region $\left[\epsilon_{I}^{(\text {core })}=0\right]$, and, for the figure at the right, gain is present in the core $\left[\epsilon_{I}{ }^{(\text {core })}=\epsilon_{I}{ }^{(\mathrm{clad})}\right]$. 
Table 1. Calculated Parameters, with and without Core Gain

\begin{tabular}{|c|c|c|c|c|}
\hline \multirow[b]{3}{*}{ Parameter } & \multicolumn{2}{|c|}{ First Structure } & \multicolumn{2}{|c|}{ Second Structure } \\
\hline & With & Without & With & Without \\
\hline & Core Gain & Core Gain & Core Gain & Core Gain \\
\hline Cladding width (each section), $L(\mu \mathrm{m})$ & 5 & 5 & 15 & 15 \\
\hline Core width, $W(\mu \mathrm{m})$ & 0.578 & 0.265 & 0.126 & 0.055 \\
\hline Gain constant, $\beta_{I}\left(\mathrm{~cm}^{-1}\right)$ & 72.7 & 58.7 & 74.4 & 73.3 \\
\hline Detuning, $\nu$ & -0.053 & 0.124 & 0.183 & 0.199 \\
\hline Gain enhancement, $\eta$ & 1.015 & 0.818 & 1.038 & 1.023 \\
\hline Power leakage (dB) & -35.8 & -26.8 & -47.2 & -31.2 \\
\hline
\end{tabular}

Equation (15) is the main result of this analysis. It can be written in terms of magnitude and phase as

$$
\begin{aligned}
\exp \left\{\left[\frac{\beta_{R} \beta_{I}}{k_{0}}-\frac{\omega^{2} \mu \epsilon_{I}{ }^{(\text {core })}}{2 k_{0}}\right] W\right\} & \\
= & \left|\frac{\kappa}{(\gamma-i \Delta k)-S \operatorname{coth}(S L)}\right|, \\
2 k_{0} W & =\operatorname{Phase}\left[\frac{ \pm \kappa}{(\gamma-i \Delta k)-S \operatorname{coth}(S L)}\right] .
\end{aligned}
$$

We use Eq. (17) to determine the core width. In the case of a passive triangular lattice and $\Delta k=0$, Phase $(\kappa)=-\pi / 2$, and, as $k_{0}=\pi / b$, the allowed values of $W$ are $b / 4,5 b / 4, \ldots$ for even-symmetry modes and $3 b / 4,7 b / 4, \ldots$ for odd-symmetry modes. ${ }^{5}$ The latter require the use of the - in Eq. (12). In the remainder of this Letter we concentrate on the even-symmetric lowest-order solution.

The full transverse profile is given by

$$
E_{\perp}(x)=\left\{\begin{array}{ll}
E_{\perp}{ }^{(\text {clad })}(x) & 0<x \leq L \\
E^{(\text {core })}(x) & -W \leq x \leq 0 \\
E_{\perp}{ }^{(\text {clad })}(-x+W) & -(L+W) \leq x<-W
\end{array} .\right.
$$

The modal equations, Eqs. (16) and (17), both involve gain constant $\beta_{I}$ and core width $W$ in a complicated way, which requires a self-consistent numerical solution. We analyze two structures: the first with cladding width $L=5 \mu \mathrm{m}$ and the second with cladding width $L=15 \mu \mathrm{m}$.

For $L=5 \mu \mathrm{m}$, Fig. 2 shows the transverse mode profiles when $\epsilon_{I}{ }^{\text {(core) }}=0$ and when $\epsilon_{I}{ }^{\text {(core })}=\epsilon_{I}$ (clad) . The Bloch nature of the field in the cladding is clearly evident. We use the following numerical values: wavelength, $\lambda_{0}=1.55 \mu \mathrm{m}$; real part of the dielectric coefficient, $\epsilon_{R}=12.5 \epsilon_{0}$; imaginary part of the dielectric coefficient, $\epsilon_{I}=10^{-3} \epsilon_{R}$; strength of the grating, $\epsilon_{1}=\epsilon_{R} / 40$; and transverse periodicity of the cladding, $b=1.25 \times 1.55 \mu \mathrm{m}$. The calculated numerical values of the gain and core width, etc. in all four cases are listed in Table 1 . Detuning $\nu$ is defined by the relationship $k_{0}=2(1+\nu) \pi / b$ and, in most cases, the optimum operating point is not exactly on resonance when $\nu \equiv 0$. Power leakage refers to the field intensity at the outer edge of the cladding; although the field component propagating toward the core is taken to be zero by our boundary condition $B(L)=0$ in solving Eq. (8), the outward-propagating component $A(L)$ is typically not zero. As Table 1 shows, however, we can make it small by increasing the width of the cladding region. For wider cladding (greater $L$ ), the envelope has to decay more slowly with $x$ to satisfy $B(L)=0$; core width $W$ is forced to be smaller, so the field does not decay so much away from the center peak.

Gain enhancement $\eta$ refers to the increase of $\beta_{I}$ in this structure compared with the gain constant of bulk dielectric. It is an enhancement of the exponential factor, and the typical increase is a substantial advantage of these structures compared with conventional uniform-dielectric waveguides. This enhancement reflects the fact that in a transverse Bragg resonance laser the wave spends much more time in the cladding than in the core. Greater gain may be achieved, or shorter devices will suffice for given gain. Also, the substantial field content outside the narrow core region leads to a larger modal cross-sectional area, and consequently to higher output power, than for conventional semiconductor laser structures. The field extends further into the cladding in the absence of core gain $\left[\epsilon_{I}{ }^{(\text {core })}=0\right]$, indicating a trade-off between overall power output and longitudinal gain enhancement factor $\eta$.

Lang et $a l .{ }^{7}$ have considered broad area lasers, which are confined by transverse passive Bragg mirrors. Gain in the Bragg claddings was not considered.

The authors thank John M. Choi, Bruno Crosignani, William Green, Reginald K. Lee, Paolo Di Porto, George T. Paloczi, and R. Leheny for useful discussions. This study was funded by the U.S. Office of Naval Research (Y. S. Park) and the U.S. Air Force Office of Scientific Research (H. Schlossberg). A. Yariv's e-mail address is ayariv@caltech.edu.

\section{References}

1. H. Stoll and A. Yariv, Opt. Commun. 8, 5 (1973).

2. P. Yeh and A. Yariv, Opt. Commun. 19, 427 (1976).

3. M. Nakamura, A. Yariv, H. W. Yen, and S. Somekh, Appl. Phys. Lett. 22, 515 (1973).

4. A. Yariv and H. W. Yen, Opt. Commun. 10, 120 (1974).

5. A. Yariv, Opt. Lett. 27, 936 (2002).

6. A. Yariv, Optical Electronics in Modern Communications, 5th ed. (Oxford U. Press, Oxford, 1997).

7. R. J. Lang, K. Dzurko, A. A. Hardy, S. Demars, A. Schoenfelder, and D. F. Welch, IEEE J. Quantum Electron. 34, 2196 (1998). 